\title{
Evidence for Structural Variants of a- and b-Type Peptide Fragment Ions Using Combined Ion Mobility/Mass Spectrometry
}

\author{
Isabel Riba-Garcia, ${ }^{a}$ Kevin Giles, ${ }^{\mathrm{b}}$ Robert H. Bateman, ${ }^{\mathrm{b}}$ and \\ Simon J. Gaskell ${ }^{\mathrm{a}}$ \\ ${ }^{a}$ Michael Barber Centre for Mass Spectrometry, School of Chemistry and Manchester Interdisciplinary \\ Biocentre, University of Manchester, Manchester, United Kingdom \\ ${ }^{\mathrm{b}}$ Waters MS Technologies Centre, Manchester, United Kingdom
}

\begin{abstract}
Tandem mass spectrometry (MS/MS) of peptides plays a key role in the field of proteomics, and an understanding of the fragmentation mechanisms involved is vital for data interpretation. Not all the fragment ions observed by low-energy collision-induced dissociation of protonated peptides are readily explained by the generally accepted structures for a- and b-ions. The possibility of a macrocyclic structure for b-type ions has been recently proposed. In this study, we have undertaken investigations of linear protonated YAGFL- $\mathrm{NH}_{2}, \mathrm{~N}$-acetylated-YAGFL- $\mathrm{NH}_{2}$, and cyclo-(YAGFL) peptides and their fragments using a combination of ion mobility (IM) separation and mass spectrometry. The use of IM in this work both gives insight into relative structural forms of the ion species and crucial separation of isobaric species. Our study provides compelling evidence for the formation of a stable macrocyclic structure for the $b_{5}$ ion generated by fragmentation of protonated linear YAGFL- $\mathrm{NH}_{2}$. Additionally we demonstrate that the $\mathrm{a}_{4}$ ion fragment of protonated YAGFL- $\mathrm{NH}_{2}$ has at least two structures; one of which is attributable to a macrocyclic structure on the basis of its subsequent fragmentation. More generally, this work emphasizes the value of combined IM-MS/MS in probing the detailed fragmentation mechanisms of peptide ions, and illustrates the use of combined ion mobility/ collisional activation/mass spectrometry analysis in achieving an effective enhancement of the resolution of the mobility separator. (J Am Soc Mass Spectrom 2008, 19, 609-613) (C 2008 American Society for Mass Spectrometry
\end{abstract}

$\mathrm{T}$ andem mass spectrometric (MS/MS) characterization of peptides has become a central analytical approach to the analysis of the proteomes of cells and organisms [1]. Accordingly, it is important to understand the gas-phase ion chemistry that underpins the MS/MS of peptide ions in order to optimize the application of the technique. The structures of the $\mathrm{N}$-terminal $\mathrm{a}-$ and $\mathrm{b}$-ions formed from the fragmentation of collisionally activated protonated peptides in the gas phase [2] have been a topic of investigation and discussion for several years. The b-ions have generally been considered to consist of a linear peptide chain terminating in a cyclic oxazolone structure (Scheme 1a) [3]; subsequent formation of a-ions by loss of $\mathrm{CO}$ has been presumed to follow opening of the oxazolone ring. These hypotheses alone, however, do not readily explain all the fragment ions observed in low-energy collision-induced dissociation experiments. Yagüe et al. [4] proposed rearrangement of b-ions to give macrocyclic intermediate structures that could fragment further to yield products in more complete agreement with

Address reprint requests to Professor Simon J. Gaskell, Michael Barber Centre for Mass Spectrometry, School of Chemistry and Manchester Interdisciplinary Biocentre, University of Manchester, 131 Princess St., Manchester M1 7DN, UK. E-mail: Simon.Gaskell@manchester.ac.uk observed data. In earlier work, Tang and Boyd [5] reported evidence of an interaction between the primary amine groups of lysine or ornithine residues with the C-terminus of peptide ions. Recently, Harrison et al. [6] used both computational studies and comparison of the breakdown graphs of the $b_{5}$ ion (produced by fragmentation of protonated YAGFL- $\mathrm{NH}_{2}$ ) and the protonated cyclo-(YAGFL) peptide analogue to provide strong evidence for a cyclic structure for the $b_{5}$-ion moiety (Scheme 1b). Current ideas [4, 7-9] suggest a mixture of possible linear and cyclic structures for a-ions.

To gain further insight into the possible structures of the $a-$ and b-ions, information on the relative size of each ion species would be of value, to complement characterization by mass/charge ratio. Such information is attainable using ion mobility (IM) spectrometry in which ion species are driven through a background neutral gas under the influence of an electric field, and separate according to their differing mobilities. The mobility of a particular ion species is dependent on factors such as the charge state and mass, but more importantly on the interaction cross-section with the background gas. Thus, there is the potential to separate different structures of the isobaric a-ions and b-ions 
(a)<smiles>[R]C(N)C(=O)NC([R2])C(=O)NC([R3])C(=O)NC([R4])C1=[NH+]C([R5])C(=O)O1</smiles>

(b)

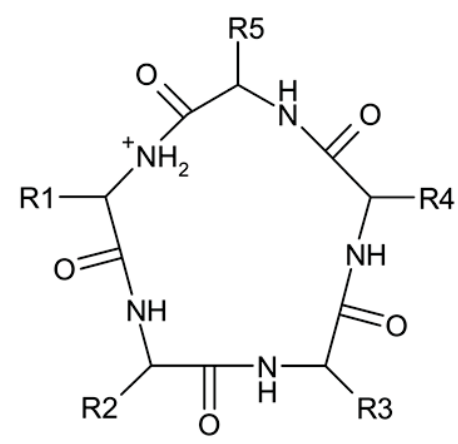

Scheme 1. Proposed (a) oxazolone and (b) macrocyclic structures for $b_{5}$ ions.

using this technique. Through the combination of IM-MS and IM-MS/MS analysis of the linear YAGFL$\mathrm{NH}_{2}$ peptide, we provide here further evidence consistent with a stable macrocyclic structure of b-ions and describe new evidence for the structural variety of a-ions.

\section{Experimental}

The linear YAGFL- $\mathrm{NH}_{2}$ peptide was obtained from Bachem Biosciences, King of Prussia, PA and cycloYAGFL peptide from Celtek Peptides, Nashville, TN. The $\mathrm{N}$-acetylated YAGFL- $\mathrm{NH}_{2}$ peptide was synthesized by incubation of linear YAGFL- $\mathrm{NH}_{2}$ with $50 \mu \mathrm{L}$ of acetic anhydride for $30 \mathrm{~min}$. The resulting peptide was desalted using a peptide Trap cartridge (Michrom BioResources, Auburn, CA). All samples were diluted to a concentration of $0.5 \mathrm{pmol} / \mu \mathrm{L}$ using $50 / 50$ acetonitrile/water $0.1 \%$ formic acid and infused at a flow rate of $5 \mu \mathrm{L} / \mathrm{min}$ into the electrospray ionization source of a Waters Synapt HDMS instrument (Waters Corp., Manchester, UK) for ion mobility and mass spectrometric analysis. The Synapt instrument has a hybrid quadrupole/ion mobility/ orthogonal acceleration time-of-flight (OA-TOF) geometry [10]. The ion mobility separation stage of the Synapt comprises of three ion guides: the first (Trap) is used to accumulate ions before mobility separation; the second (IM) performs the ion mobility separation and the third (Transfer) transports the mobility-separated ions to the OA-TOF for mass analysis. Ion mobility separation is achieved using a continual sequence of traveling voltage waves (T-Waves) to propel ions through a background gas; the drag due to the presence of gas causes ions to periodically slip over the waves, with ions of higher mobility slipping over less often than those of lower mobility and so exiting the device first [10]. Ion mobility spectra are recorded by synchro- nizing the OA TOF mass spectrometric acquisitions with the gated release of ions from the Trap ion guide into the IM separation guide. For these experiments, ions were released over a $100 \mu$ s period into the IM guide and the arrival time distributions (ATDs) of the mobility separated ions recorded in 200 sequential mass spectra ( $\equiv 13 \mathrm{~ms}$ total time). The IM guide was operated at a pressure of 0.5 mbar of nitrogen and the Trap and Transfer guides operated at a pressure of $\sim 0.03 \mathrm{mbar}$ ( 1:1 vol/vol nitrogen:argon). The IM guide T-Wave was operated at $300 \mathrm{~m} / \mathrm{s}$ and linearly ramped in amplitude from 5 to $20 \mathrm{~V}$ over each $13 \mathrm{~ms}$ experiment. The Transfer guide T-Wave was operated at $247 \mathrm{~m} / \mathrm{s}$ and with a constant $3 \mathrm{~V}$ amplitude. The resolution of ion mobility separations $(\mathrm{t} / \Delta \mathrm{t})$ was $\sim 8-10$.

For IM acquisitions with no fragmentation, ion injection voltages into the Trap and Transfer were set at 5 and $3 \mathrm{~V}$, respectively. Ion fragmentation pre- or post-IM separation was achieved by increasing the Trap or Transfer ion injection voltages, respectively; values used are given in the Results and Discussion section.

Instrument control and data acquisition were performed using MassLynx V4.1 software (Waters Corp., Manchester, UK).

\section{Results and Discussion}

The linear YAGFL- $\mathrm{NH}_{2}(\mathrm{M}+\mathrm{H})^{+}$ion at $\mathrm{m} / \mathrm{z} 569.2$ was selected using the quadrupole and injected into the Trap guide with $22 \mathrm{eV}$ to induce fragmentation before mobility separation. Figure 1 shows arrival time distributions (ATDs) for the $(\mathrm{M}+\mathrm{H})^{+}$ion of YAGFL- $\mathrm{NH}_{2}$ and the mobility separated fragment ions derived from this precursor. Figure 1a shows all such fragments in a plot of $\mathrm{m} / \mathrm{z}$ versus arrival time with color-coding of signal intensities. Figure $1 \mathrm{~b}$ shows extracted mobility plots for selected $m / z$ species. The ATD for the $b_{5}$ fragment ion $(m / z 552.2)$ is displaced to a shorter time $(5.40 \mathrm{~ms})$ in comparison with that of the precursor ion ( $\mathrm{m} / z$ 569.2, $5.59 \mathrm{~ms})$. The small mass difference between these two species is expected to have a negligible effect on their relative ion mobilities so that, as both species are singly charged, the difference in mobility is largely attributable to structural effects. Also shown in Figure $1 \mathrm{~b}$ is the ATD for the $(\mathrm{M}+\mathrm{H})^{+}$ion of cyclo-(YAGFL) $(m / z 552.2)$ derived from a separate experiment (under nonfragmenting conditions). The shorter ATD of the cyclo-(YAGFL) $(\mathrm{M}+\mathrm{H})^{+}$ion $(5.35 \mathrm{~ms})$ compared with that of the linear YAGFL- $\mathrm{NH}_{2}$ ion $(5.59 \mathrm{~ms})$ is consistent with the cyclo-(YAGFL) ion having a smaller collision cross-section (more compact structure) than the linear ion, as has been previously shown by Ruotolo et al. [11]. Replicate experiments (data not shown) indicated high reproducibility with the difference between ATDs recorded for the same ion in separate experiments being much less than the difference between the ATDs of the linear and cyclic peptide ions. That the ATD for the $b_{5}$ ion is significantly closer to that of the cyclo-(YAGFL) $(\mathrm{M}+\mathrm{H})^{+}$ion than the linear YAGFL-NH $\mathrm{NH}_{2}(\mathrm{M}+\mathrm{H})^{+}$ 


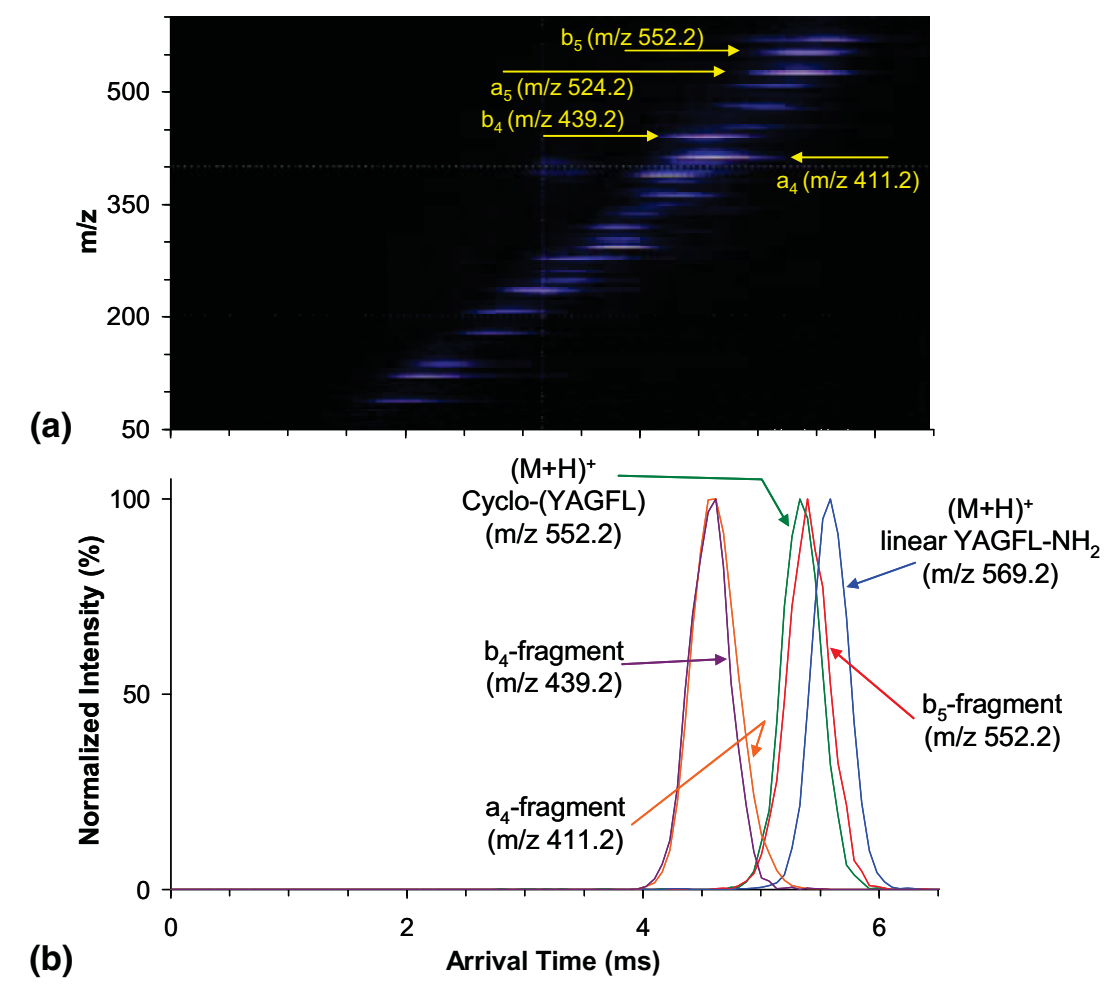

Figure 1. Arrival time distributions for the $(\mathrm{M}+\mathrm{H})^{+}$ion of YAGFL- $\mathrm{NH}_{2}$ and fragment ions derived from this precursor. (a) Plot of arrival time versus $\mathrm{m} / \mathrm{z}$ with color-coding of signal intensities; (b) Extracted arrival time distributions of selected $\mathrm{m} / \mathrm{z}$ species from $(\mathbf{a})$ together with that for the $(\mathrm{M}+\mathrm{H})^{+}$ ion of cyclo-(YAGFL).

ion, indicates the $b_{5}$ ion to possess a compact, possibly macrocyclic, structure. This observation supports the assertion of Harrison et al. [6] that this $b_{5}$-ion can exist in cyclic form, which serves as a precursor for indirect second-generation fragment ions. The mobility data reported here, however, go further in suggesting that the compact form is stable at least on a millisecond time scale.

In a different experiment, the $m / z$-selected $b_{5}$ ion (generated by cone voltage fragmentation of the linear YAGFL- $\mathrm{NH}_{2}$ peptide ion) and the $(\mathrm{M}+\mathrm{H})^{+}$ion of cyclo-(YAGFL) were further compared by collisioninduced fragmentation ( $22 \mathrm{eV}$ injection energy into the Trap guide) and mobility separation of the product ions. No differences were observed between the ATDs or relative signal intensities of equivalent fragment ions from the two precursor species (see Supplementary information, which can be found in the electronic version of this article).

The linear $\mathrm{N}$-acetylated YAGFL- $\mathrm{NH}_{2}$ peptide was also analyzed. Fragmentation of the $(\mathrm{M}+\mathrm{H})^{+}$ion of this species cannot produce the proposed $b_{5}$-macrocyclic structure seen in Scheme $\mathbf{1 b}$, due to the lack of free amino group at the $\mathrm{N}$-terminus, but is expected to form the oxazolone species (Scheme 1a). Figure 2 shows the ATDs for the $(\mathrm{M}+\mathrm{H})^{+}$ion $(\mathrm{m} / z$ 611.2, $5.74 \mathrm{~ms})$ and for the $\mathrm{N}$-acetylated $\mathrm{b}_{5}$ ion $(\mathrm{m} / \mathrm{z} 594.2,5.73 \mathrm{~ms})$; the ATDs for these species are indistinguishable, indicating that the $b_{5}$ oxazolone moiety has a similar collision cross- section to the linear precursor peptide, in contrast to the linear YAGFL- $\mathrm{NH}_{2}(\mathrm{M}+\mathrm{H})^{+}$and $b_{5}$ fragment ion pair (Figure 1b). This result further substantiates the proposed hypothesis for a compact (macrocyclic) fragment $\mathrm{b}_{5}$ ion from the linear YAGFL- $\mathrm{NH}_{2}$ precursor ion.

Further significant observations from Figure 1 include a difference in the ATDs of the $a_{4}$ and $b_{4}$ ions [12]. Close inspection of the ATD for the $a_{4}$ fragment reveals it to be somewhat broader than for the $b_{4}$ ion, suggesting the presence of multiple structures. This would be consistent with recent suggestions [9]. The mobility resolution, however, is not sufficient to separate discrete components. To investigate the $a_{4}$ ion species further, we induced fragmentation of this ion in the Transfer guide region of the Synapt instrument, i.e., postmobility separation. Using this approach, precursor ions and their corresponding fragment ions can be assigned through correlation of respective ATDs.

The $a_{4}$ ion species were generated by cone voltage fragmentation of the $(\mathrm{M}+\mathrm{H})^{+}$ion of linear YAGFL$\mathrm{NH}_{2}$, selected using the quadrupole mass filter and injected with low-energy into the Trap ion guide. The $\mathrm{a}_{4}$ species then underwent mobility separation and were fragmented using $26 \mathrm{eV}$ injection energy into the Transfer guide. Figure 3 shows the ATDs derived from the second-generation fragment ions at $\mathrm{m} / \mathrm{z} 309.2$ and 207.1 together with that for the $\mathbf{a}_{4}$ precursor ion $(\mathrm{m} / z$ 411.2). The ATD derived from $m / z 207.1$ has essentially the same overall broad profile as that of the $\mathrm{a}_{4}$ precursor ion 


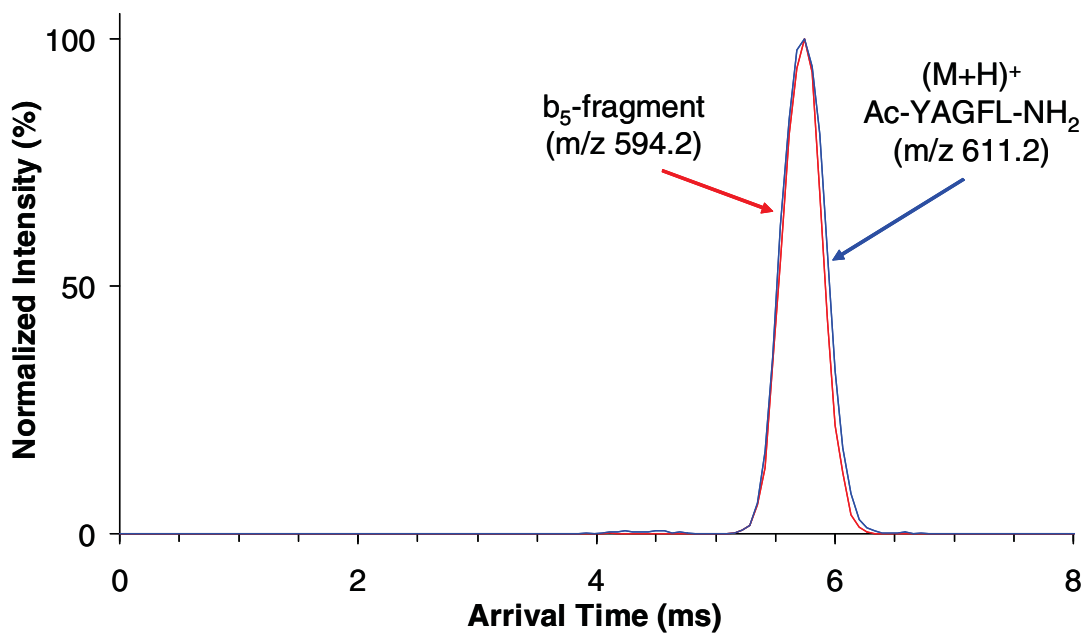

Figure 2. Arrival time distributions for the $(\mathrm{M}+\mathrm{H})^{+}$ion of $\mathrm{N}$-acetylated-YAGFL- $\mathrm{NH}_{2}$ and the $\mathrm{N}$-acetylated $b_{5}$ fragment ion generated from this precursor ( $15 \mathrm{eV}$ injection energy into the Trap guide).

species (both centered on $4.63 \mathrm{~ms}$ ), whereas that derived from $\mathrm{m} / \mathrm{z} 309.2$ is shifted towards the leading edge of the precursor peak (centered on $4.50 \mathrm{~ms}$ ) and is notably narrower. Since the ATDs of the second generation ion species reflect those of their respective precursor ions, these data indicate the presence of multiple forms of the $\mathrm{a}_{4}$ precursor ions. Interestingly, the $\mathrm{m} / \mathrm{z}$ 309.2 ion ([YAF-28-17-28 $+\mathrm{H}]^{+}$) can only be formed from a cyclic $\mathrm{a}_{4}$ precursor ion whereas the $\mathrm{m} / \mathrm{z} 207.1$ ion $\left([\mathrm{YA}-28+\mathrm{H}]^{+}\right.$) can be formed from a cyclic or linear $\mathrm{a}_{4}$ structure. Detection of other fragment ions which can be formed from either a linear or cyclic precursor (e.g., $m / z 292.1\left(\left[Y A G+\mathrm{H}^{+}\right)\right.$, $235.1\left([\mathrm{YA}+\mathrm{H}]^{+}\right)$, or $177.1\left([\mathrm{GF}-28+\mathrm{H}]^{+}\right)$) yield the same ATD profile as $\mathrm{m} / \mathrm{z}$ 207.1, and those that require a cyclic precursor, e.g., $\mathrm{m} / \mathrm{z}$ $337.2\left([Y A F-28-17+\mathrm{H}]^{+}\right)$, yield the same ATD profile as $m / z 309.2$ (data not shown). Figure 4 shows the mass

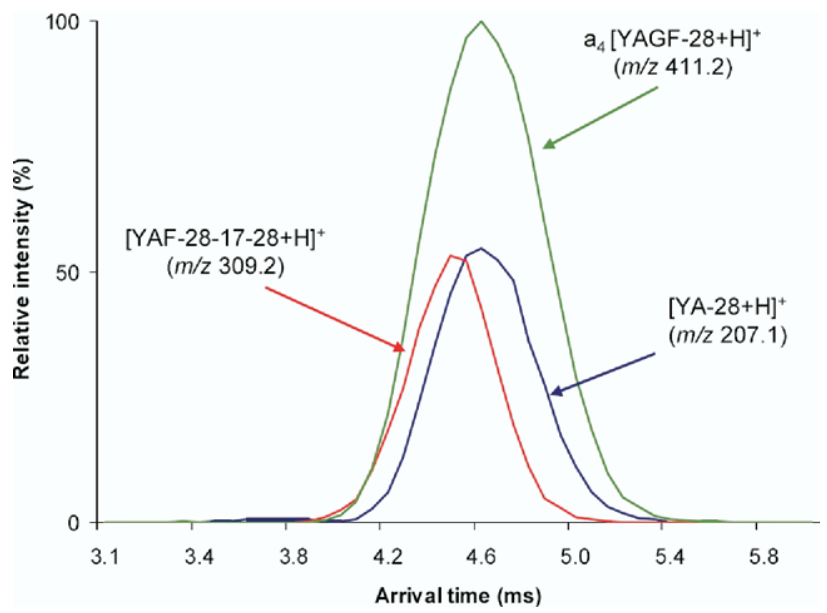

Figure 3. Arrival time distributions derived from detection of $\mathrm{a}_{4}$ ions $(\mathrm{m} / \mathrm{z} 411.2)$ and from detection of second-generation fragment ions ([YAF-28-17-28 $+\mathrm{H}]^{+}, m / z$ 309.2) and [YA-28 $\left.+\mathrm{H}\right]^{+}, m / z$ 207.1) formed post-IM separation. spectra obtained from the leading and trailing edge of the ATD obtained from post-mobility fragmentation of the $a_{4}$ ion. The two mass spectra are qualitatively similar; however, the intensities and distribution of the fragment ions in the different parts of the ATD highlight that a subset of product ions form from different structures. Since the resolution of the mobility device is not sufficient to completely separate the precursor structures, some overlap of the fragment ions is inevitable. Overall, the post-IM fragmentation results are consistent with the presence of a heterogeneous population of $\mathrm{a}_{4}$ ions (with lifetimes at least of the order of milliseconds), including both linear and cyclic structures, and are in accord with a recent study where cyclic and linear $\mathrm{a}_{4}$ ions were identified using infra-red spectroscopy [9]. The capability for collision-induced fragmentation of ions after mobility separation provides the general capability of interrogating an ion population through a mobility distribution [13]. This provides the potential for achieving an increase in the effective resolution of the ion mobility separation. We note the conceptual parallel between this approach and that advanced many years ago in using mass spectrometric detection to enhance the effective resolution of gas chromatographic separations [14].

Equivalent post-IM fragmentation experiments of the $b_{4}, b_{5}$, and $a_{5}$ ion species also provide preliminary evidence for a mixture of structures for each ion (data not shown). However, in these instances the differences in ATD derived from fragments of putatively rearranged and non-rearranged precursor ions are not as significant as in the $a_{4}$ ion experiment, and further investigations are in progress.

\section{Conclusions}

In summary, our study provides further evidence consistent with the formation of a stable compact, macro- 


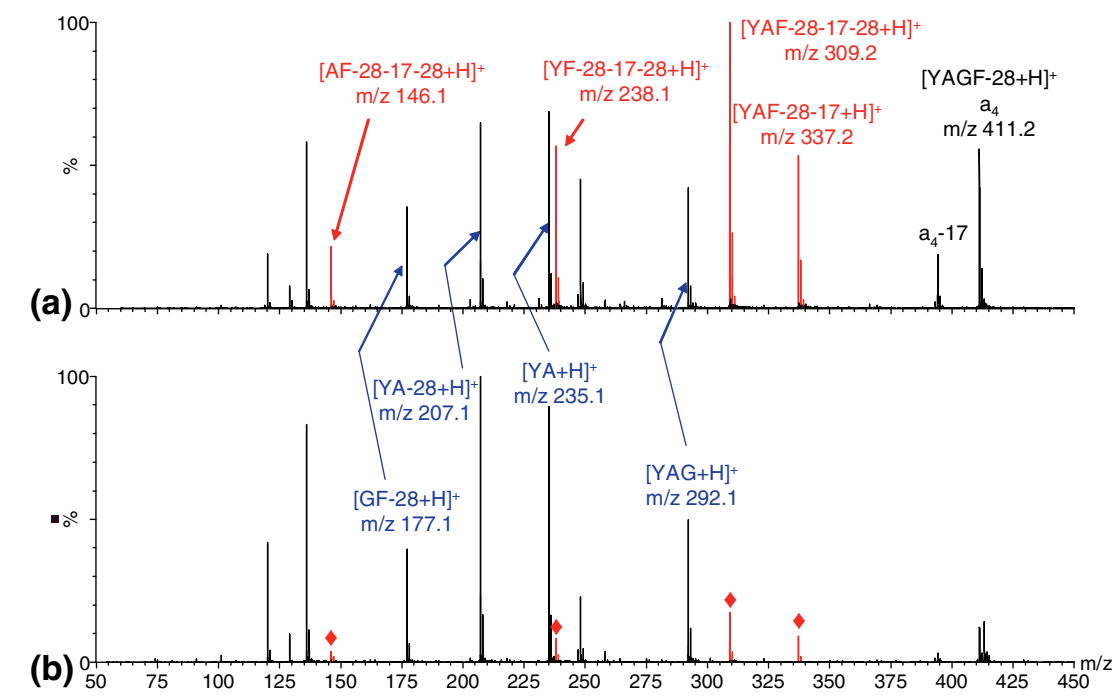

Figure 4. Product ion spectra corresponding to (a) leading and (b) trailing edges of the ATD profile from fragmentation of the $\mathrm{a}_{4}$ ion after mobility separation (filled diamond indicates those fragments which can only be obtained from a cyclic precursor).

cyclic, structure for the $b_{5}$ ion generated by fragmentation of protonated linear YAGFL- $\mathrm{NH}_{2}$. Additionally, we have demonstrated that the $\mathrm{a}_{4}$ ion fragment of protonated YAGFL- $\mathrm{NH}_{2}$ has at least two structures; that of higher mobility (i.e., smaller interaction cross-section) is attributed to a macrocyclic structure and is shown to give rise specifically to second generation fragment ions consistent only with a cyclic precursor. More generally, this work confirms the value of combined IM-MS and IM-MS/MS in probing the detailed fragmentation mechanisms of peptide ions. Of particular value is the potential for providing an effective increase in mobility resolution through fragmentation of ions post-mobility separation.

\section{Acknowledgments}

The authors thank Professor Vicki Wysocki (Department of Chemistry, University of Arizona), Dr. Alex Harrison (Department of Chemistry, University of Toronto), and Dr. Bela Paizs (German Cancer Research Center, Heidelberg) for kindly providing the YAGFL-NH ${ }_{2}$ and cyclo-(YAGFL) samples. They thank BBSRC for financial support (grants EP/D013615/1 and IIP0307/005).

\section{References}

1. Aebersold, R.; Mann, M. Mass Spectrometry-Based Proteomics. Nature 2003, 422, 198-207.

2. Biemann, K. Contributions of Mass Spectrometry to Peptide and Protein Structure. Biomed. Environ. Mass Spectrom. 1988, 16, 99-111.
3. Yalcin, T.; Khouw, C.; Csizmadia, I. G.; Peterson, M. R.; Harrison, A. G. Why are b-Ions Stable Species in Peptide Spectra?. J. Am. Soc. Mass Spectrom. 1995, 6, 1165-1174.

4. Yagüe, J.; Paradela, A.; Ramos, M.; Ogueta, S.; Marina, A.; Barahona, F.; Lopez de Castro, J. A.; Vazquez, J. Peptide Rearrangement During Quadrupole Ion Trap Fragmentation: Added Complexity to MS/MS Spectra. Anal. Chem. 2003, 75, 1524-1535.

5. Tang, X. J.; Boyd, R. K. Rearrangements of Doubly Charged Acylium Ions From Lysyl and Ornithyl Peptides. Rapid Commun. Mass Spectrom. 1994, 8, 678-686.

6. Harrison, A. G.; Young, A. B.; Bleiholder, C.; Suhai, S.; Paizs, B. Scrambling of Sequence Information in Collision-Induced Dissociation of Peptides. J. Am. Chem. Soc. 2006, 128, 10364-10365.

7. Paizs, B.; Suhai, S. Fragmentation Pathways of Protonated Peptides. Mass Spectrom. Rev. 2005, 24, 508-548.

8. Jia C.; Qi W.; He Z. Cyclization Reaction of Peptide Fragment Ions During Multistage Collisionally Activated Decomposition: An Inducement to Lose Internal Amino-Acid Residues. J. Am. Soc. Mass Spectrom. 2007, 18, 663-678.

9. Polfer, N. C.; Oomens, J.; Suhai, S.; Paizs, B. Infrared Spectroscopy and Theoretical Studies on Gas-Phase Protonated Leu-Enkephalin and Its Fragments: Direct Experimental Evidence for the Mobile Proton. J. Am. Chem. Soc. 2007, 129, 5887-5897.

10. Pringle, S. D.; Giles, K.; Wildgoose, J. L.; Slade, S. E.; Thalassinos, K.; Bateman, R. H.; Bowers, M. T.; Scrivens, J. H. An Investigation of the Mobility Separation of Some Peptide and Protein Ions Using a New Hybrid Quadrupole/Traveling Wave IMS/OA-TOF Instrument. Int. J. Mass Spectrom. 2007, 261, 1-12.

11. Ruotolo, B. T.; Tate, C. C.; Russell, D. H. Ion Mobility-Mass Spectrometry Applied to Cyclic Peptide Analysis: Conformational Preferences of Gramicidin S and Linear Analogs in the Gas Phase. J. Am. Soc. Mass Spectrom. 2004, 15, 870-878.

12. Riba-Garcia, I.; Giles, K.; Gaskell, S. J. A Contribution of Ion Mobility Mass Spectrometry to the Investigation of Peptide Fragment Ion Structure; Proceedings of the 55th ASMS Conference on Mass Spectrometry; Indianapolis, IN, Month of conference was May/June, 2007.

13. Merenbloom, S. I.; Koeniger, S. L.; Valentine, S. J.; Plasencia, M. D.; Clemmer, D. E. IMS-IMS and IMS-IMS-IMS/MS for Separating Peptide and Protein Fragment Ions. Anal. Chem. 2006, 78, 2802-2809.

14. Biller, J. E.; Biemann, K. Reconstructed Mass Spectra, a Novel Approach for the Utilization of Gas Chromatograph-Mass Spectrometer Data. Anal. Lett. 1974, 7, 515-528. 